\title{
A Comparative Study between Implicit and Crank-Nicolson Finite Difference Method for Option Pricing
}

\author{
Tanmoy Kumar Debnath ${ }^{1, *}$, A B M Shahadat Hossain ${ }^{2}$ \\ ${ }^{1}$ Department of Business Administration, Shanto-Mariam University of Creative Technology, Dhaka-1230, \\ Bangladesh \\ ${ }^{2}$ Department of Applied Mathematics, University of Dhaka, Dhaka-1000, Bangladesh
}

\begin{abstract}
In this paper, we have applied the finite difference methods (FDMs) for the valuation of European put option (EPO). We have mainly focused the application of Implicit finite difference method (IFDM) and CrankNicolson finite difference method (CNFDM) for option pricing. Both these techniques are used to discretized Black-Scholes (BS) partial differential equation (PDE). We have also compared the convergence of the IFDM and CNFDM to the analytic BS price of the option. This turns out a conclusion that both these techniques are fairly fruitful and excellent for option pricing.
\end{abstract}

(C) 2020 Published by Bangladesh Mathematical Society

Received: 16 March, 2020 Accepted: 09 July, 2020

Keywords: European put option; Black-Scholes equation; Implicit finite difference method; Crank-Nicolson finite difference method.

AMS Subject Classification 2010: 65M06; 65B15.

\section{Introduction}

Option pricing plays a very significant role in a stock market. By the help of option contract the seller and buyer can hedge their losses in stock market. So it enticed the attention of innumerable empiricists. In short, Option

*Corresponding author: Tanmoy Kumar Debnath, E-mail address: tanmoymathdu@ gmail.com 
is a contract which gives the buyer (the owner or holder of the option) the right, but not an obligation, to buy or sell an underlying asset at a specified strike price on or before a specified date, depending on the form of the option. There are two types of option namely, call option and put option. Every option has two types of positions, namely, a long position and a short position. Options can be either American or European. FDMs are effectively applicable to all kinds of call and put option. Here we focused only EPO. Put option gives the holder the right to sell the underlying asset by a certain date for a certain price [1]. Based on their exercised conditions European options can be exercised only on the expiration date itself, while American options can be exercised at any time up to the expiration date [1].

BS model is a well-known tool for pricing different kinds of options which is developed by Fischer Black, Myron Scholes and Robert Merton in the early 1970s. This model also known as Black-Scholes-Merton model [1]. However, BS model always leads to a direct numerical calculation of the price of option. The BS equation is a PDE which relies on two independent variables. These two variables are the time and the stock price which follows a random path. FDM is computationally efficient and useful to solve PDEs and provide a general numerical solution to the valuation problems, as well as an optimal early exercise strategy. FDMs calculate the value of a derivative by solving the differential equation that the derivative satisfies. The differential equation is converted into a set of difference equations and that difference equations are solved iteratively.

In this paper, we will try to show the comparative robustness between the IFDM and CNFDM for pricing European options.

\section{Literature Review}

Numerical methods have been applied to the different field of mathematics and it allure the attention of many researchers of several kinds of field. Numerical solutions play a very important role in the field of computational finance. It has been applied for the pricing of various types of options such as European, American, Asian and many other types of options. Schwartz [2] first used FDMs to option pricing. A closed form solution first proposed by Merton [3]. Fadugba and Nwozo [4] presented the application of CNFDM for the valuation of options. They intended to show in their paper the accuracy, convergence and stability of CNFDM for the valuation of European options. Kumar et al. [5] presented the comparison between analytic and numerical solutions of linear BS equation governing option pricing using BANKNIFTY. Courtadon [6] introduced a model based on a finite difference approximation of the equation of option valuation and standard numerical methods. Recently Nwobi et al. [10] presented the impact of CNFDM in valuations of options. In their paper they tried to identify the sources of mispricing in order to reduce pricing bias. Anwar and Andallah [11] presented the comparison between explicit FDM and semi-IFDM for option pricing and discussed the stability of the explicit FDM.

The outline of this paper is organized as follows. At first we represent the methodology of option pricing, discretization of the BS PDE, boundary conditions, FDMs such as Implicit and Crank-Nicolson scheme for numerically solving the price of the option. Then we provide the example of numerical solutions obtained by the implementing the algorithms with MATLAB which are converges to the BS analytic solutions and show the comparison between IFDM and CNFDM. Finally, we will conclude that which method is more efficient and which method takes more time for evaluating option price. 


\section{Methodology}

Many option contract values can be obtained by solving PDEs with certain initial and boundary conditions. The FDM is one of the premier mathematical tool employed to solve PDEs. The Black, Scholes and Merton showed that a riskless portfolio made up of an asset with value $S$ and an option value $f(t, S)$ satisfy the following the partial differential equation.

$$
\frac{\partial f(t, S)}{\partial t}+r S \frac{\partial f(t, S)}{\partial S}+\frac{1}{2} S^{2} \sigma^{2} \frac{\partial^{2} f(t, S)}{\partial S^{2}}=r f(t, S)
$$

where $f$ is the price of option as a well-defined function of time $t$ and stock price $S$ [12], $r$ is the risk-free interest rate and $\sigma$ is the volatility of the stock. This equation is known as BS PDE.

Equation (3.1) has many solutions, corresponding to all the different derivatives that can be defined with $S$ as the underlying variable. The particular derivatives can be obtained by solving BS PDE which depends on boundary conditions [1]. The boundary conditions specify the values of the derivative at the boundaries of possible values of $S$ and $t$. In the case of European put option, the key boundary condition is

$$
f=\max (K-S, 0) \quad \text { when } t=T \text {. }
$$

\subsection{BS option pricing formulas}

The most famous solutions to the BS PDE (3.1) are the Black-Scholes-Merton formulas for the price of European call and put options. These formulas are:

$$
\begin{aligned}
& c=N\left(d_{1}\right) S-N\left(d_{2}\right) K e^{-r T} \\
& p=N\left(-d_{2}\right) K e^{-r T}-N\left(-d_{1}\right) S,
\end{aligned}
$$

where

$$
\begin{aligned}
d_{1} & =\frac{1}{\sigma \sqrt{T}}\left[\ln \left(\frac{S}{K}\right)+T\left(r+\frac{\sigma^{2}}{2}\right)\right] \\
d_{2} & =\frac{1}{\sigma \sqrt{T}}\left[\ln \left(\frac{S}{K}\right)+T\left(r-\frac{\sigma^{2}}{2}\right)\right] \\
& =d_{1}-\sigma \sqrt{T},
\end{aligned}
$$

where $c$ is Call premium, $p$ is Put premium, $S$ is Current stock price or Asset or Spot price, $t$ is Option expiration, $K$ is Strike price, $r$ is Risk-free interest rate, $N$ is Cumulative standard normal distribution. 


\subsection{Discretization of the BS PDE}

We discretize the equation (3.1) with respect to time, $t$ and to the underlying asset price, $S$. Divide the $(t, S)$ plane into a sufficiently thick grid or mesh, and approximate in tiny steps $\Delta S$ and $\Delta t$ by some small fixed finite steps. We define an array of $N+1$ equally spaced grid points $t_{0}, t_{1}, \ldots, t_{N}$ to discretize the time derivative with $t_{i+1}-t_{i}=\Delta t$ and $\Delta t=T / N$.

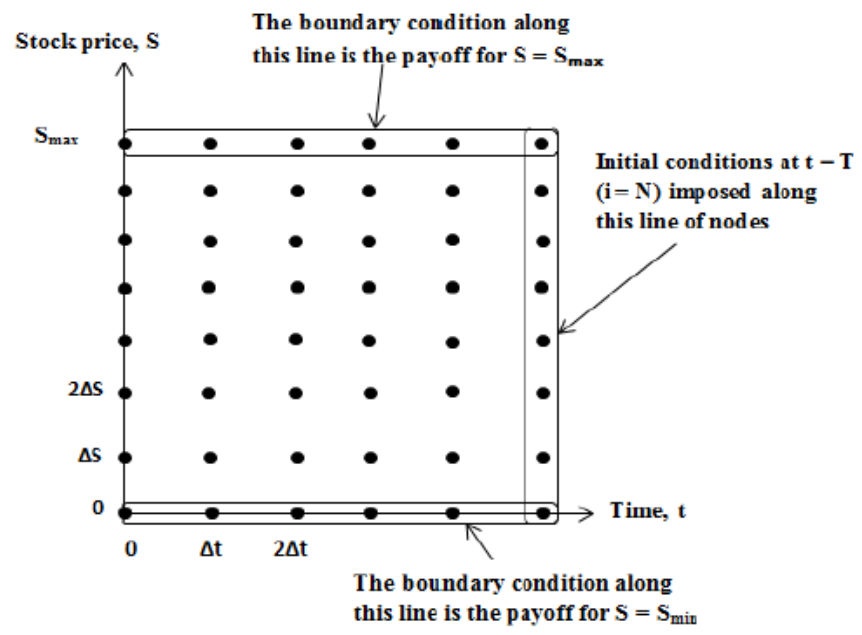

Fig.3.1. The mesh points for the finite difference approximation

We know that the stock price cannot go below 0 and maximum stock price can be signified by $S_{\text {max }}$. We have $M+1$ equally spaced grid points $S_{0}, S_{1}, \ldots, S_{M}$ to discretize the stock price derivative with $S_{j+1}-S_{j}=\Delta S$ and $\Delta S=S_{\max } / M$.

We denote the value of the derivative at time step $t_{i}$ when the underlying asset has value $S_{j}$ as

$$
f_{i, j}=f(i \Delta t, j \Delta S)=f\left(t_{i}, S_{j}\right)=f(t, S),
$$

where $i$ and $j$ are the number of discrete increments in the time to maturity and stock price respectively, $\Delta t$ and $\Delta S$ are the discrete increments in the time to maturity and the stock prices. The quantities $f_{N, j}$ for $j=0,1,2, \ldots, M, f_{i, 0}$ and $f_{i, M}$ for $i=0,1, \ldots, N$ are referred to as the boundary values which may or may not be known ahead of time but in our PDE they are known. The quantities $f_{i, j}$ for $i=0,1, \ldots, N-1$ and 
$j=1,2, \ldots, M-1$ are referred to as interior points or values [7].

\subsection{Boundary Conditions}

In order to solve Equation (3.1) at time instant $i \Delta t$ we need to obtain the option values at:

- The upper asset boundary

- The lower asset boundary

- The initial values that are specified at option maturity.

The European put involve the PDE on the domain $S \in[0, \infty]$. This presents a difficulty. We must represent this range by a finite set of points. A reasonable fix is to truncate the domain to $S \in[0, M]$, where $M$ is some suitably large value [9].

We will first reiterate our grid and boundary conditions:

Discretized time: $0, \Delta t, 2 \Delta t, \ldots, N \Delta t ; \quad$ where $N \Delta t=T$

Discretized price: $0, \Delta S, 2 \Delta S, \ldots, M \Delta S$; where $M \Delta S=S_{\max }$

In Fig.3.1, at the upper asset boundary, we consider $j=M$. So $M \Delta S=S_{\max }$.

For the value of the European put option is:

$$
f_{i, M}=0, \quad i=0,1, \ldots, N
$$

At the lower asset boundary, $j=0$, so the value of the $j \Delta S$ is zero. So the payoff at expiration will be $K$ and discounting back to time $t$, we have the value of the European put options is:

$$
f_{i, 0}=K e^{-r(N-i) \Delta t}, \quad i=0,1, \ldots, N
$$

Now for the boundary values at option maturity, $i=N$, the initial option (boundary) values of the European put option is:

$$
f_{N, j}=\max (K-j \Delta S, 0), \quad j=0,1, \ldots, M
$$

Equations (3.6), (3.7) and (3.8) define the values of the European put option along the three edges of the grid in the Fig. 3.1, where $S=S_{\max }, S=0$ and $t=T$. 
Table 3.1: Boundary conditions for EPO

\begin{tabular}{cc}
\hline Boundary & Put Option \\
\hline$t=T$ & $f_{N, j}=\max (K-j \Delta S, 0)$ \\
$S=S_{\max }$ & $f_{i, M}=0$ \\
$S=0$ & $f_{i, 0}=K e^{-r(N-i) \Delta t}$ \\
\end{tabular}

\subsection{Finite Difference Approximation}

We approximate the derivative with respect to time, $t$ by a backward difference and approximate the derivative with respect to, $S$ by a central difference. This is not the only possibility, but any choice must be somehow compatible with the boundary conditions [8].

Consider that the option price denoted by $f(t, S)$ for an interior point $(i, j)$ on the grid. The expansion of $f(t, S+\Delta S)$ in Taylor's series can be approximated as

$$
\frac{\partial f}{\partial S}=\frac{f_{i, j+1}-f_{i, j}}{\Delta S} .
$$

This equation is known as first forward difference approximation of $\frac{\partial f}{\partial S}$ with first order accuracy. The expansion of $f(t, S-\Delta S)$ in Taylor's series can be approximated as

$$
\frac{\partial f}{\partial S}=\frac{f_{i, j}-f_{i, j-1}}{\Delta S} .
$$

This equation is known as first backward difference approximation of $\frac{\partial f}{\partial S}$ with first order accuracy.

The first order partial derivative results in the central difference given by

$$
\frac{\partial f}{\partial S}=\frac{f_{i, j+1}-f_{i, j-1}}{2 \Delta S} .
$$

The second order partial derivatives can be estimated by the symmetric central difference approximation. which can be approximated as 


$$
\frac{\partial^{2} f}{\partial S^{2}}=\frac{f_{i, j+1}-2 f_{i, j}+f_{i, j-1}}{\Delta S^{2}}
$$

Expand $f(t+\Delta t, S)$ in Taylor's series we get

$$
\frac{\partial f}{\partial t}=\frac{f_{i+1, j}-f_{i, j}}{\Delta t}
$$

which is known as first forward difference approximation of $\frac{\partial f}{\partial t}$ with first order accuracy. The BS PDE converts into a difference equation and will be solved iteratively for using to approximate the solution $f(t, S)$.

\subsection{Discretized by Implicit Scheme}

Substituting Equations (3.11), (3.12) and (3.13) into the BS PDE (3.1) and noting that $S=j \Delta S$ and rearranging terms we obtain,

$$
\begin{aligned}
& a_{j} f_{i, j-1}+b_{j} f_{i, j}+c_{j} f_{i, j+1}=f_{i+1, j}, \\
& \quad \text { for } j=1,2, \ldots, M-1 \text { and } i=0,1, \ldots, N-1,
\end{aligned}
$$

where

$$
\begin{aligned}
& a_{j}=\frac{1}{2} r j \Delta t-\frac{1}{2} \sigma^{2} j^{2} \Delta t \\
& b_{j}=1+\sigma^{2} j^{2} \Delta t+r \Delta t \\
& c_{j}=-\frac{1}{2} r j \Delta t-\frac{1}{2} \sigma^{2} j^{2} \Delta t .
\end{aligned}
$$

Here we express $f_{i+1, j}$ implicitly in-terms of the unknowns $f_{i, j-1}, f_{i, j}$ and $f_{i, j+1}$. Implicit method has accuracy up to $O\left(\Delta t, \Delta S^{2}\right)$.

\subsection{Discretized by Crank-Nicolson Scheme}

Now the goal is to discretize the BS PDE (3.1). So we use a central approximation for $\frac{\partial f}{\partial t}$ at the point $f_{i-1 / 2, j}:$ 


$$
\frac{\partial f_{i-1 / 2, j}}{\partial t}=\frac{f_{i, j}-f_{i-1, j}}{\Delta t}+O\left(\Delta t^{2}\right)
$$

We use a central approximation for $\frac{\partial f}{\partial S}$ at the point $f_{i-1 / 2, j}$ :

$$
\begin{aligned}
\frac{\partial f_{i-1 / 2, j}}{\partial S} & =\frac{1}{2}\left[\frac{\partial f_{i-1, j}}{\partial S}+\frac{\partial f_{i, j}}{\partial S}\right] \\
= & \frac{1}{2}\left[\frac{f_{i-1, j+1}-f_{i-1, j-1}}{2 \Delta S}+\frac{f_{i, j+1}-f_{i, j-1}}{2 \Delta S}\right]+O\left(\Delta S^{2}\right) .
\end{aligned}
$$

We use a standard approximation for $\frac{\partial^{2} f}{\partial S^{2}}$ at the point $f_{i-1 / 2, j}$ :

$$
\begin{aligned}
\frac{\partial^{2} f_{i-1 / 2, j}}{\partial S^{2}} & =\frac{1}{2}\left[\frac{\partial^{2} f_{i-1, j}}{\partial S^{2}}+\frac{\partial^{2} f_{i, j}}{\partial S^{2}}\right] \\
= & \frac{1}{2}\left[\frac{f_{i-1, j+1}-2 f_{i-1, j}+f_{i-1, j-1}}{\Delta S^{2}}+\frac{f_{i, j+1}-2 f_{i, j}+f_{i, j-1}}{\Delta S^{2}}\right]+O\left(\Delta S^{2}\right) .
\end{aligned}
$$

Substituting these approximations into the BS PDE (3.1) we get,

$$
\begin{array}{r}
-a_{j}^{\prime} f_{i-1, j-1}+\left(1-b_{j}^{\prime}\right) f_{i-1, j}-c_{j}^{\prime} f_{i-1, j+1}=a_{j}^{\prime} f_{i, j-1}+\left(1+b_{j}^{\prime}\right) f_{i, j}+c_{j}^{\prime} f_{i, j+1}, \\
\text { for } j=1,2, \ldots, M-1 \text { and } i=N, \ldots, 1,
\end{array}
$$

where

$$
\begin{aligned}
& a_{j}^{\prime}=\frac{\Delta t}{4}\left(\sigma^{2} j^{2}-r j\right) ; \\
& b_{j}^{\prime}=-\frac{\Delta t}{2}\left(\sigma^{2} j^{2}+r\right) ; \\
& c_{j}^{\prime}=\frac{\Delta t}{4}\left(\sigma^{2} j^{2}+r j\right) .
\end{aligned}
$$

CNFDM has accuracy up to $O\left(\Delta t^{2}, \Delta S^{2}\right)$. 


\section{Numerical Examples and Results}

We will calculate the price of a EPO on a non-dividend paying stock when the stock price is $\$ 69$, the strike price is $\$ 70$, the risk-free interest rate is $5 \%$ per annum, the volatility is $35 \%$ per annum, and the time to maturity is 6 months.

By the IFDM MATLAB implementation, the numerical approximate result of EPO price is $\$ 6.3997$. By the BS put option pricing formula (3.3) the analytic solution (theoretical result) is $\$ 6.4014$. By the CNFDM MATLAB implementation, the numerical approximate result of EPO price is $\$ 6.4014$. We can see that in the case of CNFDM the numerical result coincides with the BS analytic result corrected to four decimal places.

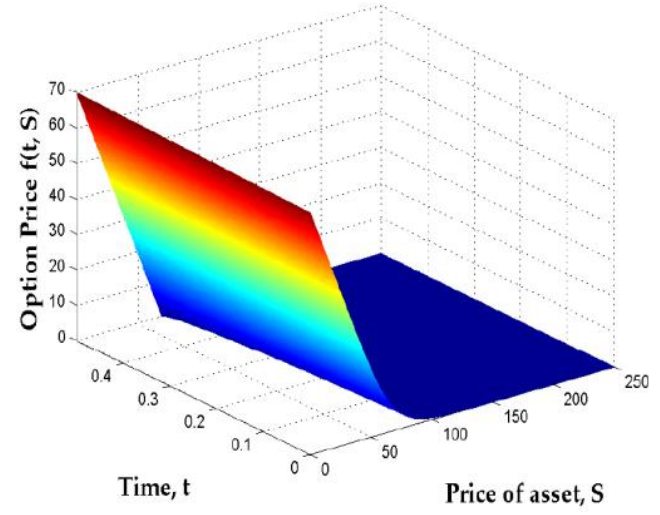

(a)

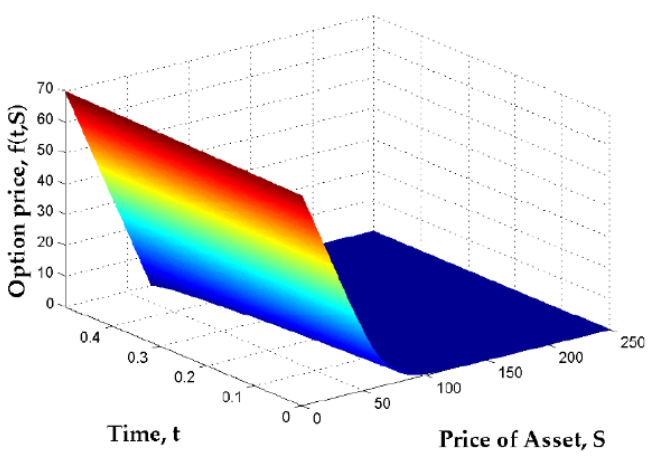

(b)

Fig.4.1. (a) 3-D plot of European put option price by IFDM; (b) 3-D plot of European put option price by CNFDM.

From Fig.4.1 we can see that both the numerical method gives fairly accurate results. In MATLAB code we set asset grid points, $M=600$ and time grid points, $N=500$. We might try to improve them by using finer grid. Now we will see that what will be happened when the time grid $N$ and the stock price grid $M$ increases in the case of when $M$ equal to $N$ and $M$ not equal to $N$.

Table 4.1: The results of IFDM when $M=N$. Here $K=\$ 70, S=\$ 69, r=0.05, \sigma=0.35, \mathrm{~T}=0.5, S_{\max }=$ 250.

\begin{tabular}{cccc}
\hline $\mathrm{M}=\mathrm{N}$ & European put & BS result & Absolute Error \\
\hline 5 & 12.7776 & 6.4014 & 6.3762 \\
10 & 7.3281 & 6.4014 & 0.9267 \\
15 & 6.3219 & 6.4014 & 0.0795 \\
20 & 6.8261 & 6.4014 & 0.4247 \\
25 & 6.1772 & 6.4014 & 0.2242 \\
30 & 6.5214 & 6.4014 & 0.1200 \\
35 & 6.4633 & 6.4014 & 0.0619 \\
\hline
\end{tabular}




\begin{tabular}{clll}
\hline 40 & 6.3533 & 6.4014 & 0.0481 \\
45 & 6.4662 & 6.4014 & 0.0648 \\
50 & 6.3605 & 6.4014 & 0.0409 \\
55 & 6.4174 & 6.4014 & 0.0160 \\
60 & 6.4196 & 6.4014 & 0.0182 \\
65 & 6.3824 & 6.4014 & 0.0190 \\
70 & 6.4196 & 6.4014 & 0.0182 \\
75 & 6.3858 & 6.4014 & 0.0156 \\
80 & 6.3975 & 6.4014 & 0.0039 \\
85 & 6.4074 & 6.4014 & 0.0060 \\
90 & 6.3960 & 6.4014 & 0.0054 \\
95 & 6.4051 & 6.4014 & 0.0037 \\
100 & 6.3926 & 6.4014 & 0.0088 \\
\hline
\end{tabular}

Table 4.2: The results of CNFDM when $M=N$. Here $K=\$ 70, S=\$ 69, r=0.05$,

$$
\sigma=0.35, \mathrm{~T}=0.5, S_{\max }=250 .
$$

\begin{tabular}{cccc}
\hline $\mathrm{M}=\mathrm{N}$ & European Put & BS result & Absolute Error \\
\hline 5 & 12.7882 & 6.4014 & 6.3868 \\
10 & 7.3746 & 6.4014 & 0.9732 \\
15 & 6.3703 & 6.4014 & 0.0311 \\
20 & 6.8580 & 6.4014 & 0.4566 \\
25 & 6.2168 & 6.4014 & 0.1846 \\
30 & 6.5483 & 6.4014 & 0.1469 \\
35 & 6.4870 & 6.4014 & 0.0856 \\
40 & 6.3753 & 6.4014 & 0.0261 \\
45 & 6.4847 & 6.4014 & 0.0833 \\
50 & 6.3780 & 6.4014 & 0.0234 \\
55 & 6.4329 & 6.4014 & 0.0315 \\
60 & 6.4338 & 6.4014 & 0.0324 \\
65 & 6.3957 & 6.4014 & 0.0057 \\
70 & 6.4317 & 6.4014 & 0.0303 \\
75 & 6.3974 & 6.4014 & 0.0040 \\
80 & 6.4082 & 6.4014 & 0.0068 \\
85 & 6.4175 & 6.4014 & 0.0161 \\
90 & 6.4055 & 6.4014 & 0.0041 \\
95 & 6.4142 & 6.4014 & 0.0128 \\
100 & 6.4012 & 6.4014 & 0.0020 \\
\hline
\end{tabular}

Table 4.1 shows that when stock price grid $M$ and time grid $N$ are same, the IFDM scheme in Equation (3.14) performs well and is consistence when the stock price grid $M$ and time grid $N$ increases as $N \rightarrow \infty, \Delta t \rightarrow 0$ and as $M \rightarrow \infty, \Delta S \rightarrow 0$. From Table 4.2 shows that the CNFDM scheme in equation (3.15) is also performs well and is more consistence than IFDM.

We can illustrate Table 4.1 and Table 4.2 by the Fig.4.2 (a) and Fig.4.2 (b) respectively. From Fig.4.2 (a) and 
Fig.4.2 (b) we can see that, at the start in both cases the option value shows some inconsistency. In the both case, as the number of time steps increases, the amplitude of oscillations reduces and the option value converges to analytic BS solution as iterations go to infinity.

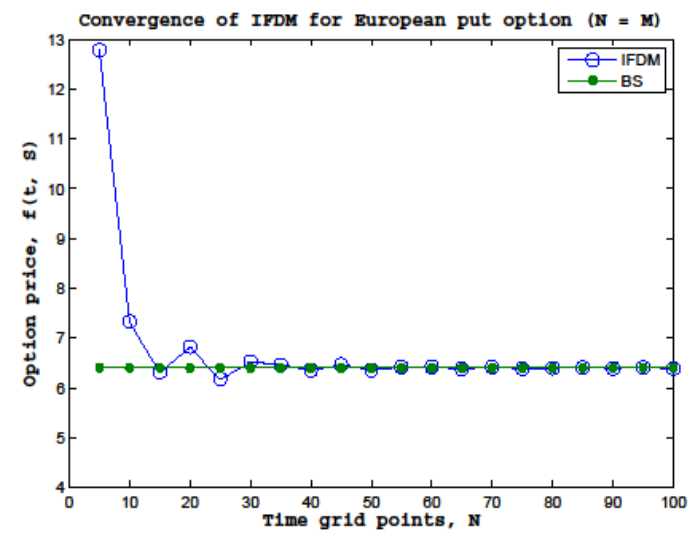

(a)

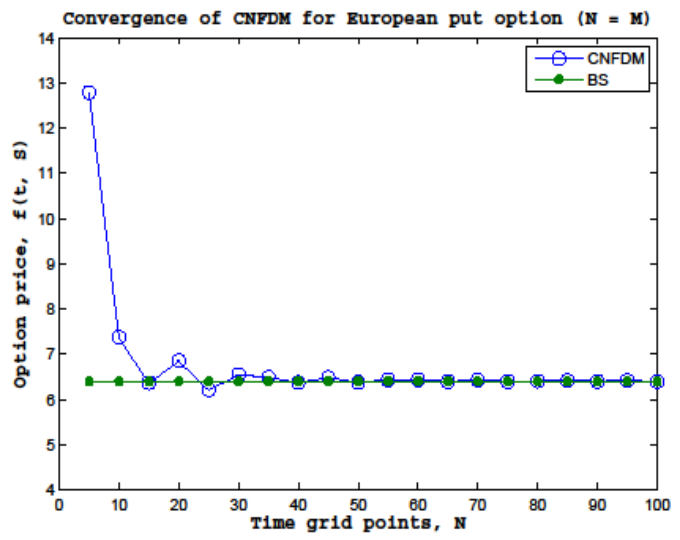

(b)

Fig.4.2. Comparison between convergence of (a) IFDM and (b) CNFDM for European put option when $N=M$ (where $S=\$ 69 ; K=\$ 70 ; r=0.05 ; \sigma=0.35 ; T=0.5)$.

Similarly, in the case of $N$ not equal to $M$, the IFDM and CNFDM both are perform well and converges faster than when the number of steps $N$ and $M$ are the same.

i.e., From Fig.4.3 (a) and Fig.4.3 (b) we can see that, in both cases at the start the option value shows less fluctuations than when the number of steps $N$ and $M$ are the same. As the number of time steps increases, in the both case the option value gradually converges to analytic BS solution.

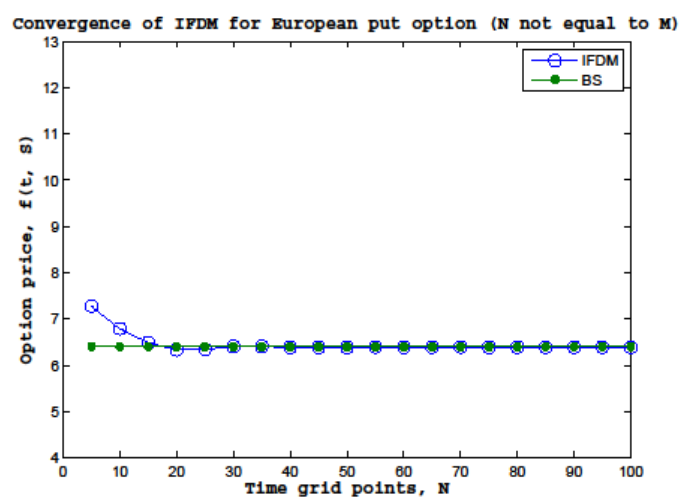

(a)

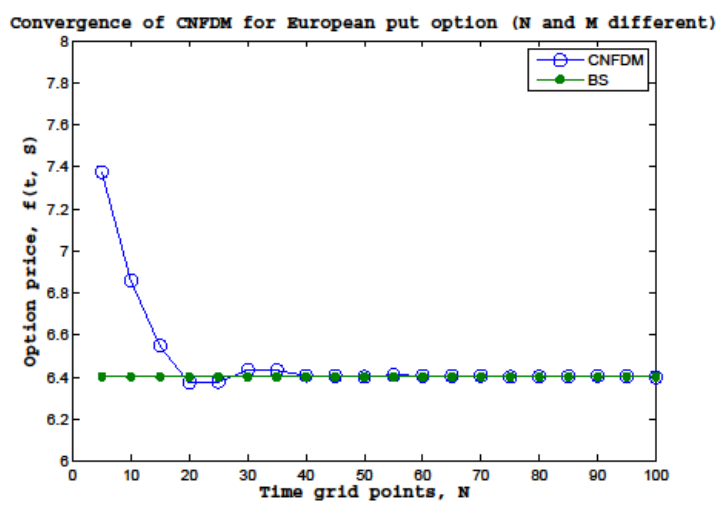

(b) 
Fig.4.3. Comparison between convergence of (a) IFDM and (b) CNFDM for European put option when $N$ and $M$ are different (where $S=\$ 69 ; K=\$ 70 ; r=0.05 ; \sigma=0.35 ; T=0.5$ )

Therefore, we conclude that when time grid, $N$ and stock price grid, $M$ are different to each other both schemes gives us good result than when time grid, $N$ equal to stock price grid, $M$.

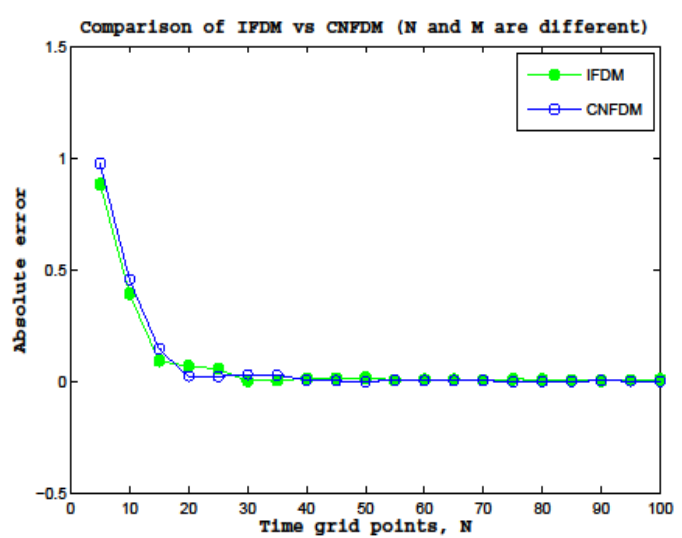

(a)

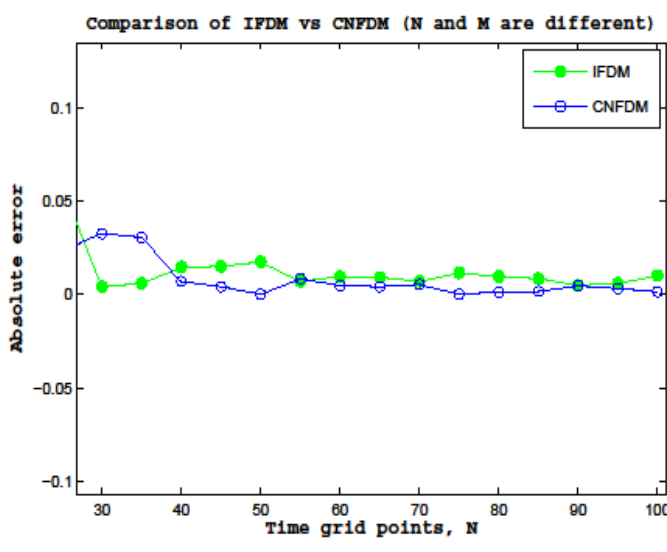

(b)

Fig.4.4. The comparison of absolute error between IFDM and CNFDM when $N$ and $M$ are different. Here (a) Normal view and (b) Zooming view

Fig.4.4 (a) shows both methods have quite similar pricing results with the BS analytic solution, which means that both methods have quite small absolute errors. More careful analysis, from Fig.4.4 (b) shows that when $N$ and $M$ increases CNFDM pricing had generate a smaller absolute error than IFDM pricing. i.e., in the both cases the CNFDM converges to BS analytic solution faster than IFDM. Thus CNFDM is more bit accurate than the IFDM.

Now we compare calculated option prices for different stock prices by different methods with the BS analytical option prices.

Table 4.3: The comparison of the absolute error IFDM vs. CNFDM for $N=M$.

Here $K=\$ 70, S=\$ 69, r=0.05, \sigma=0.35, T=0.5, N=100, M=100$.

\begin{tabular}{cccccc}
\hline S & BS result & $\begin{array}{c}\text { IFDM Result } \\
(\mathrm{N}=\mathrm{M}=100)\end{array}$ & Absolute error & $\begin{array}{c}\text { CNFDM Result } \\
(\mathrm{N}=\mathrm{M}=100)\end{array}$ & Absolut error \\
\hline 5 & 63.2717 & 63.2719 & 0.0002 & 63.2717 & 0.0000 \\
10 & 58.2717 & 58.2719 & 0.0002 & 58.2717 & 0.0000 \\
15 & 53.2717 & 53.2719 & 0.0002 & 53.2717 & 0.0000 \\
20 & 48.2717 & 48.2719 & 0.0002 & 48.2717 & 0.0000 \\
25 & 43.2717 & 43.2720 & 0.0003 & 43.2718 & 0.0001 \\
30 & 38.2730 & 38.2738 & 0.0008 & 38.2733 & 0.0003 \\
35 & 33.2844 & 33.2869 & 0.0025 & 33.2856 & 0.0012 \\
40 & 28.3420 & 28.3467 & 0.0047 & 28.3438 & 0.0018 \\
\hline
\end{tabular}




\begin{tabular}{cccccc}
\hline 45 & 23.5309 & 23.5358 & 0.0049 & 23.5316 & 0.0007 \\
50 & 18.9865 & 18.9871 & 0.0006 & 18.9834 & 0.0031 \\
55 & 14.8630 & 14.8551 & 0.0079 & 14.8543 & 0.0087 \\
60 & 11.2874 & 11.2700 & 0.0174 & 11.2735 & 0.0139 \\
65 & 8.3263 & 8.3031 & 0.0242 & 8.3093 & 0.0170 \\
70 & 5.9790 & 5.9526 & 0.0264 & 5.9615 & 0.0175 \\
75 & 4.1904 & 4.1663 & 0.0241 & 4.1747 & 0.0157 \\
80 & 2.8744 & 2.8554 & 0.0190 & 2.8618 & 0.0126 \\
85 & 1.9351 & 1.9222 & 0.0129 & 1.9259 & 0.0092 \\
90 & 1.2819 & 1.2746 & 0.0073 & 1.2757 & 0.0062 \\
95 & 0.8376 & 0.8348 & 0.0028 & 0.8338 & 0.0038 \\
100 & 0.5409 & 0.5412 & 0.0003 & 0.5389 & 0.0020 \\
\hline
\end{tabular}

Table 4.4: The comparison of the absolute error IFDM vs. CNFDM when $\mathrm{N}$ and $\mathrm{M}$ are different.

Here $K=\$ 70, S=\$ 69, r=0.05, \sigma=0.35, T=0.5, N=100, M=100$.

\begin{tabular}{cccccc}
\hline S & BS result & $\begin{array}{c}\text { IFDM Result } \\
(\mathrm{N}=100 \mathrm{M}=200)\end{array}$ & Absolute error & $\begin{array}{c}\text { CNFDM Result } \\
(\mathrm{N}=100 \mathrm{M}=200)\end{array}$ & Absolute error \\
\hline 5 & 63.2717 & 63.2719 & 0.0002 & 63.2717 & 0.0000 \\
10 & 58.2717 & 58.2719 & 0.0002 & 58.2717 & 0.0000 \\
15 & 53.2717 & 53.2719 & 0.0002 & 53.2717 & 0.0000 \\
20 & 48.2717 & 48.2719 & 0.0002 & 48.2717 & 0.0000 \\
25 & 43.2717 & 43.2720 & 0.0003 & 43.2718 & 0.0001 \\
30 & 38.2730 & 38.2736 & 0.0006 & 38.2731 & 0.0001 \\
35 & 33.2844 & 33.2861 & 0.0017 & 33.2847 & 0.0003 \\
40 & 28.3420 & 28.3454 & 0.0034 & 28.3425 & 0.0005 \\
45 & 23.5309 & 23.5353 & 0.0044 & 23.5310 & 0.0001 \\
50 & 18.9865 & 18.9893 & 0.0028 & 18.9857 & 0.0008 \\
55 & 14.8630 & 14.8615 & 0.0015 & 14.8608 & 0.0022 \\
60 & 11.2874 & 11.2804 & 0.0070 & 11.2839 & 0.0035 \\
65 & 8.3263 & 8.3150 & 0.0113 & 8.3221 & 0.0042 \\
70 & 5.9790 & 5.9658 & 0.0132 & 5.9747 & 0.0043 \\
75 & 4.1904 & 4.1781 & 0.0123 & 4.1865 & 0.0039 \\
80 & 2.8744 & 2.8649 & 0.0095 & 2.8713 & 0.0031 \\
85 & 1.9351 & 1.9291 & 0.0060 & 1.9328 & 0.0023 \\
90 & 1.2819 & 1.2793 & 0.0026 & 1.2804 & 0.0015 \\
95 & 0.8376 & 0.8376 & 0.0000 & 0.8366 & 0.0010 \\
100 & 0.5409 & 0.5427 & 0.0018 & 0.5404 & 0.0005 \\
\hline
\end{tabular}

From Table 4.3 in the case of ' $N$ equal to $M$ ', and from Table 4.4 in the case of ' $N$ not equal to $M$ ', we can see that both methods have quite similar option pricing results with the BS analytic solution, which means that both methods have approximately small absolute errors. 


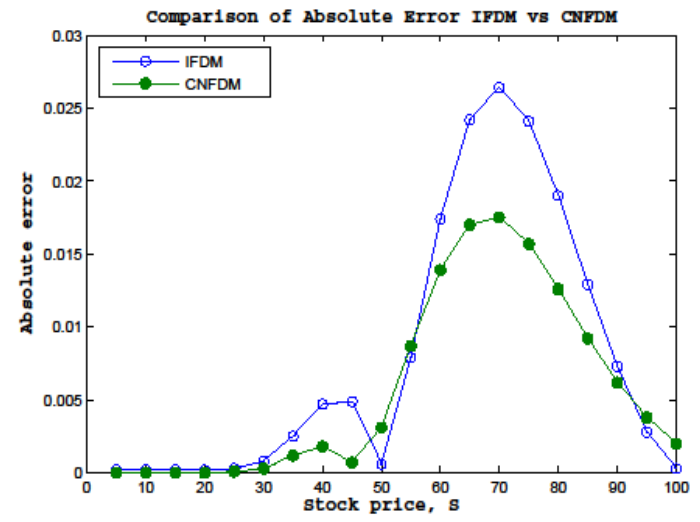

(a)

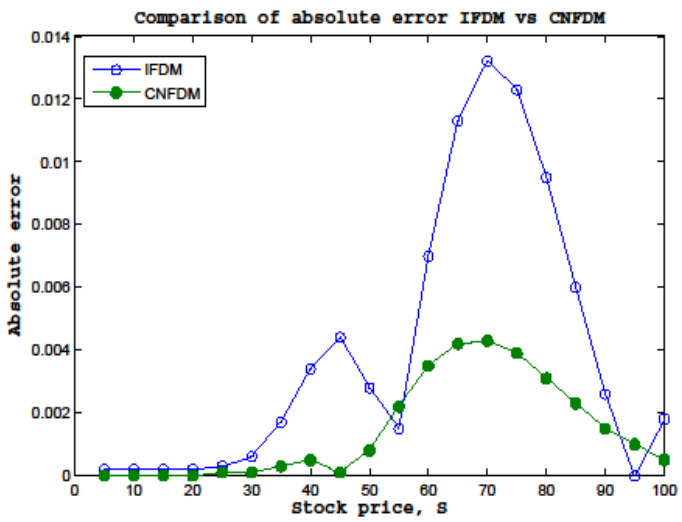

(b)

Fig.4.5. (a) Comparison of absolute error IFDM vs. CNFDM $(N=M)$; (b) Comparison of absolute error IFDM vs. CNFDM $(N \neq M)$.

From Fig.4.5 (a) and Fig.4.5 (b) we can see that, in the both cases the absolute error of CNFDM is less than the absolute error of IFDM and CNFDM is more accurate when $N$ and $M$ are different to each other.

After figure out which method is more accurate, it will be also interested and necessary to know which method is more efficient. That is to find out which method need less CPU time when pricing an option.

In graphical representation, from Fig.4.6 for ' $N=600 ; M=1200$ ' obviously, CNFDM takes more time to solve option price than IFDM. That means IFDM saves a lot more CPU-time than the CNFDM.

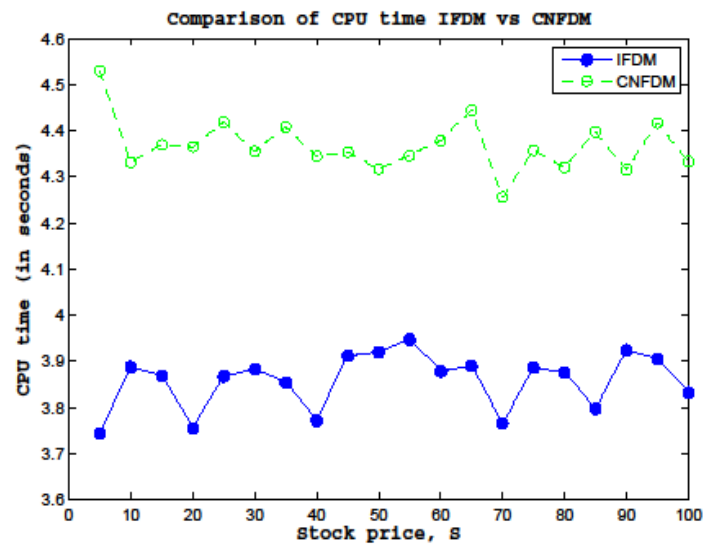

Fig.4.6. Comparison of CPU time IFDM vs. CNFDM. (where $S=\$ 69 ; K=\$ 70$;

$$
r=0.05 ; \sigma=0.35 ; T=0.5 ; N=600 ; M=1200
$$

[SAMSUNG RC418, Processor - Intel(R)Core(TM)i3 - 2310M, RAM - 2.00 GB, Hard Disk - 500 GB] 


\section{Conclusions}

Crank-Nicolson method is unconditionally stable FDM because it calculates small change in the option value for the small change of the initial conditions, converge to the solution of the PDE and calculation error decreases when the number of time and price partitions increase. The CNFDM has a higher accuracy than the IFDM so it converges faster, that means it close to the BS analytic solutions after a few iterations than IFDM. Furthermore, since CNFDM is the average of explicit and implicit FDM and it needs to solve a system of equations at each time step, so it takes more time to run. From our result we see that in the case of $N$ (time grid) not equal to $M$ (stock price grid), both methods IFDM and CNFDM converge more quickly to BS analytic solutions than when $N$ equal to $M$. CNFDM converges more quickly to the BS analytic solution than the IFDM due to its higher accuracy. That means, CNFDM is more accurate than the IFDM. Total elapsed time to run CNFDM is some more than that of IFDM. So we conclude that between IFDM and CNFDM, CNFDM is the best method and gives us more accurate result than IFDM albeit it takes more time to execute results. We use the MATLAB to get all numerical and graphical results.

\section{REFERENCES}

[1] John C. Hull, (2015-2016). Option, Futures and Other Derivatives, Global edition, Eighth edition, Pearson Education Limited.

[2] E. Schwartz, (1977). The Valuation of Warrants: Implementing a New Approach, Journal of Financial Economics, 4, 79-94.

[3] R.C. Merton, (1973). Theory of Rational Option Pricing, Bell Journal of Economics and Management Science, 4, 141-183.

[4] SE Fadugba, CR Nwozo, (2013). Crank Nicolson Finite Difference Method for the Valuation of Options, Pacific Journal of Science and Technology, 14, 136-146.

[5] M.S. Kumar, S.P. Das, M. Reza, (2012). A Comparison between Analytic and Numerical Solution of Linear Black-Scholes Equation Governing Option Pricing: Using BANKNIFTY, World Congress on Information and Communication Technologies, Trivandrum, pp.437-441.

[6] G. Courtadon, (1982). A more Accurate Finite Difference Approximation for the Valuation of Options, Journal of Financial and Quantitative Analysis, 17, 697-703.

[7] Davis Bundi Ntwiga, (2005). Numerical Methods for the Valuation of Financial Derivatives, Department of Mathematics and Applied Mathematics, University of the Western Cape, Master's thesis.

[8] Paolo Brandimarte, (2006). Numerical Methods in Finance and Economics, Second Edition, John Wiley and Sons, Inc.

[9] Desmond J. Higham, (2004). An Introduction to Financial Option Valuation, Cambridge University Press.

[10] Nwobi, Annorzie, Amadi, (2019). The Impact on Crank-Nicolson Finite Difference method in Valuation of Options, Communications in Mathematical Finance, 8(1), 93-122.

[11] Md. Nurul Anwar, Laek Sazzad Andallah, (2018). A Study on Numerical Solution of Black-Scholes Model, Journal of Mathematical Finance, 8, 372-381.

[12] M.S. Joshi, (2008). The Concepts and Practice of Mathematical Finance, Second Edition, Cambridge University Press, p. 116. 